\title{
An Optimal Reactive Power Planning Software for Urban Network
}

\author{
Qiang Sun ${ }^{1, a}$, Xue Wang ${ }^{1, b}$, Yingting $\mathrm{Ni}^{2, \mathrm{c}}$, Qiang $\mathrm{Yin}^{3, \mathrm{~d}}$, Linyu Wang ${ }^{1, \mathrm{e}}$ and \\ Fangyuan $\mathrm{Xu}^{1, \mathrm{f}}$ \\ ${ }^{1}$ State Grid Energy Research Institute, Beijing, China \\ ${ }^{2}$ Key Laboratory of Smart Grid of Ministry of Education, Tianjin University, Tianjin 300072, China \\ ${ }^{3}$ State Grid Tianjin Electric Power Corporation, Tianjin 300010, China \\ asunqiang@sgeri.sgcc.com.cn, bwangxue.xjtu@163.com, '837827077@qq.com, dtjuyin@126.com, \\ ewanglinyu@ sgeri.sgcc.com.cn, ${ }^{\mathrm{f} x u f a n g y u a n @ ~ s g e r i . s g c c . c o m . c n ~}$
}

Keywords: Reactive power planning; reactive optimization; multi-level architecture; decision-making support system; single-stage and multi-stage decomposition method.

Abstract. Considering the business features and practical requirements of the optimal reactive power planning, this paper presents an optimal reactive power planning software system for the urban network. With multi-level architecture, the software is divided into several layers, which are managed as plug-ins. Moreover, it combines single-stage planning and multi-stage planning together to find out the optimal solutions all over from the present to the target years. Based on the power network planning decision-making support system (termed CNP), the software can be embedded as a plug-in subsystem into CNP, while it can also be applied as an independent software system to achieve the tasks of reactive planning. Practical application shows that, the software is able to satisfy the practical requirements with a wide applicability and a high effectiveness.

\section{Introduction}

The urban distribution network is not only the essential part of the power system as the main power load center, but also the vital infrastructure for the urban modernization. Thus, on the basis of scientific decision, the urban network planning is ought to ensure the operating security and economy as well as the reasonability of the network modification during the load growth. Based on the load forecast, the distribution substation planning and the distribution network planning, the optimal reactive power planning (ORPP) is to determine the location and capacity of the reactive compensators to maintain the voltage levels, to decrease the network loss and consequently to realize the network security and stability in a best economic way[1]. The existing reactive optimization method is correspondingly mature $[2,3,4,5,6,7,8,9]$, but the software developed for the practical works of planning is compared to be less.

On the basis of the power network planning decision-making support system(named CNP)[10,11,12], the reactive power planning software system (RPPSS) developed in the paper can be embedded as a plug-in subsystem of CNP, while it can also be applied as an independent software system to achieve the whole tasks of reactive power planning. (CNP has been applied successfully in 500 cities or zones in China, whose function includes total load and spatial load forecast, optimal planning of $\mathrm{MV} / \mathrm{LV}$ substations, distribution network planning, reactive power planning and reliability evaluation[10].)

\section{Problems Facing Reactive Planning for Urban Network}

Optimal reactive power planning is an essential part of the urban network planning. Reasonable reactive power planning not only ensures the voltage quality and decreases the active loss, but also improves the network stability especially for the voltage stability. As is known that, the majority of the worldwide voltage collapse accidents happened in the big cities with the densely loaded receiver-side distribution network. That indicates that, to some extent, the security and stability of the network is closely connected with the allocation of reactive compensators. In the reactive power 
planning and operation, there are practical problems need to be improved, which includes the following aspects:

1) Data Measuring: Due to the deficiency of investment or other causes, the device of data measuring could not be complete, and it decreases the accuracy of power flow calculation and reactive device allocation.

2) Harmonics: At present, the shunt capacitor which is regarded as a key factor to induce harmonics is always used as a reactive compensator. Harmonics shorten the lifetime of devices, and consequently decrease the stability of the power system.

3) Reactive-power Reversing: In a condition of light load, just as the shunt capacitor is used as a reactive compensator, the reactive power may be over compensated. As a result, it may increase the reactive loss and aggravate the burden of transmission and distribution lines.

The above problems need to be considered in RPPSS.

\section{Design of the Multi-layer Reactive Optimization Software System}

Hierarchical Decomposition of Reactive Power Planning. Applied with quasi-dynamic planning method, heuristic algorithm, Bender's decomposition method, and PQ decoupled method, a general reactive planning problem is divided into a four-layer optimization. Specific as follows:

1) Multi-stage Decomposition method: Applying the Quasi-dynamic planning method, the multi-stage reactive power planning is divided into several single stages. With the data of each stage's results, the planning keeps its continuation, and consequently combines the multi-stage power planning with the reactive optimization for the target years.

2) Single-stage Decomposition Method: Firstly use the heuristic algorithm based on the sensitivity to calculate a preliminary scheme of reactive power allocation and voltage regulation. In this scheme, the system variables at different load levels should satisfy the loose operating constraints, and moreover provide a proper initial point for the latter iterative optimization. And then the method of Bender's decomposition starts.

3) Bender's Decomposition: On the basis of improved initial state, the method of Bender's decomposition is applied to decompose the problem of the single-stage and multiple load-level planning into an investment sub-problem along with several operation sub-problems. And through an alternate iteration of investment and operation sub-problems, the optimal solution can be calculated. Furthermore, the single-stage optimal result can be consequently obtained.

4) Sub-problem Decomposition: As for a certain investment scheme, the operation sub-problems are treated as the usual short range network planning (reactive power planning for the present or target years' network). In the calculation, apply the PQ decoupled algorithm and set up the power flow model to calculate the final optimal results.

The above hierarchical solving process can be briefly described as Fig. 1. (Suppose the problem involves $I$ years to plan, and $N$ load levels, $j$ and $k$ respectively represent the year $j$ and the load level k.) 


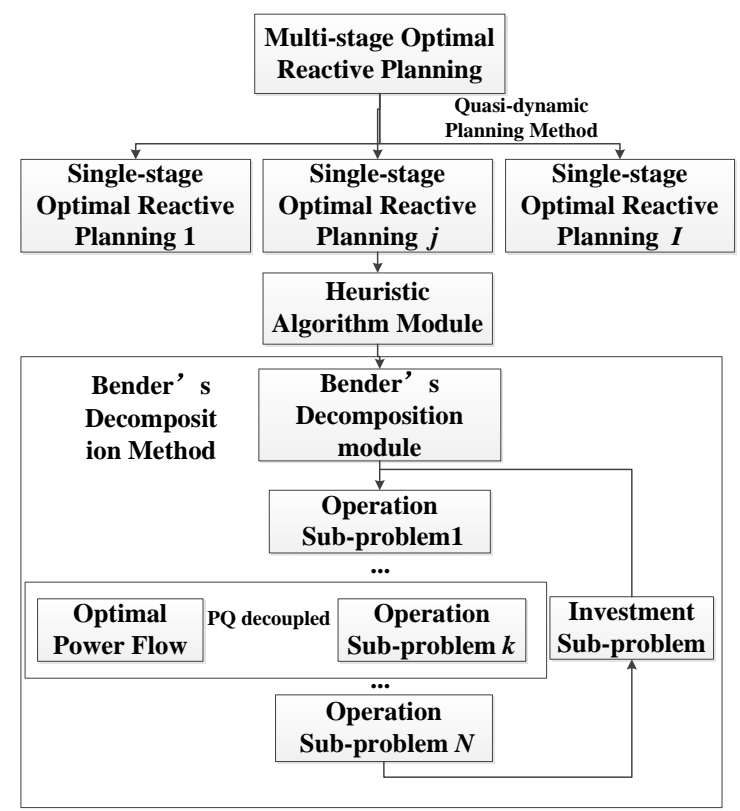

Fig. 1. Hierarchical decomposition of reactive power planning

Layered Structure of RPPSS. In order to realize the scalability and flexibility of functional reorganization, based on the common thoughts of layered architecture [11, 12], the structure of RPPSS is designed to be four layers: data layer, models and algorithm layer, application layer and interface layer. These layers are combined as plug-ins to realize the function of optimal reactive power planning. The whole structure of the software is as Fig. 2 shows:

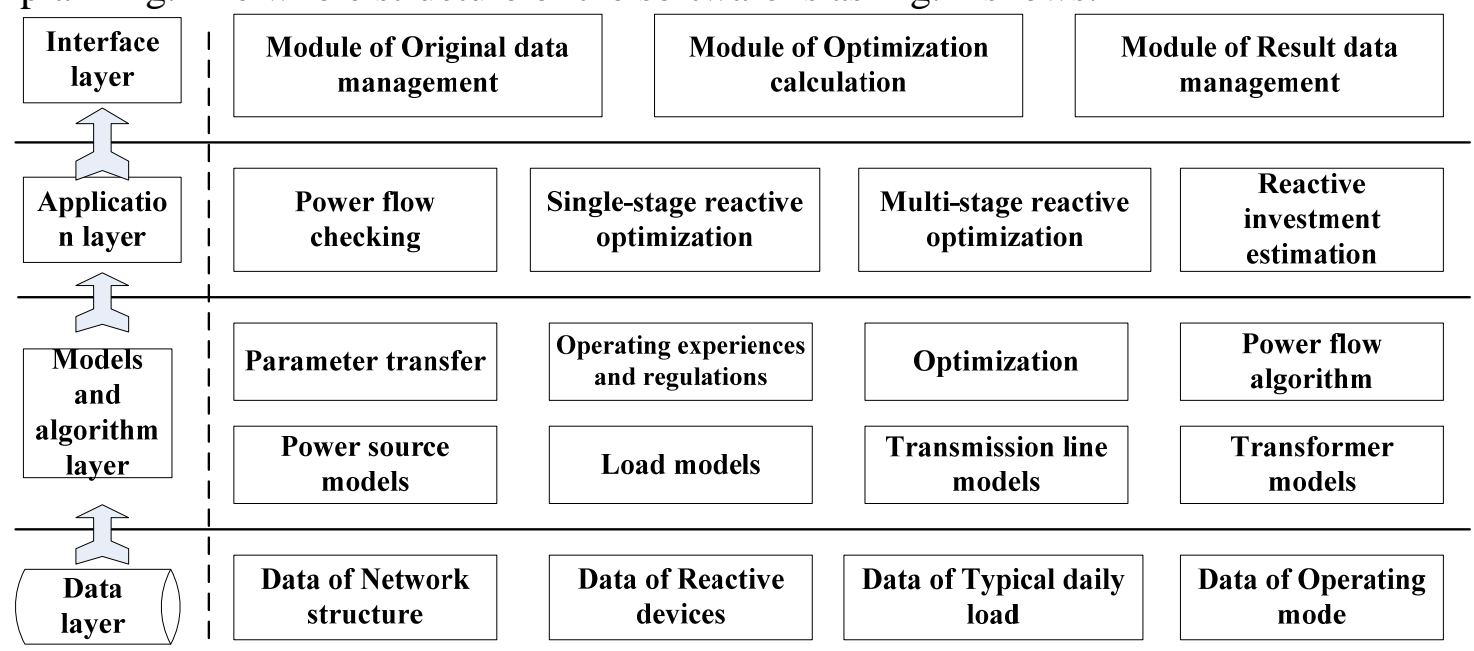

Fig. 2. Layered structures of RPPSS

The bottom data layer includes the basic data of the network: the data of network structure, the data of reactive devices, the data of typical daily load, and the data of different operating modes. These data can be either transferred from the other subsystems of CNP, or be input alone or in batch from the module of data management.

The models and algorithm layer contains the models of load, generator and transmission lines, as well as the transformation of the related parameters and the optimal method applied in the software. Meanwhile, large amounts of the operating experiences and regulation are also involved in the layer.

The application layer is specially designed for the functions of reactive optimization. By the means of calling the related algorithms through the standardized interface, the layer realizes the related functions of optimal reactive planning. The main function includes power flow calculation and checking, single-stage and multi-stage reactive optimization, as well as the reactive investment estimation.

The interface layer, which is set up on the platform of Microsoft Visual C++ and Visual Fortran, realizes the flexible dispatch of the basic data and the inner-set models and algorithms[9][11]. The layer supports the functions of the single-stage and multi-stage reactive optimization, as well as the 
result comparison and financial analysis of the multiple planning schemes. Thus, the layer can provide a visual interface for the users.

\section{Structure and Implementation of Optimal Reactive Planning Software System}

According to the function to divide, RPPSS mainly includes three parts: original data management, reactive planning calculation and result data management. The structure is shown in Fig. 3.

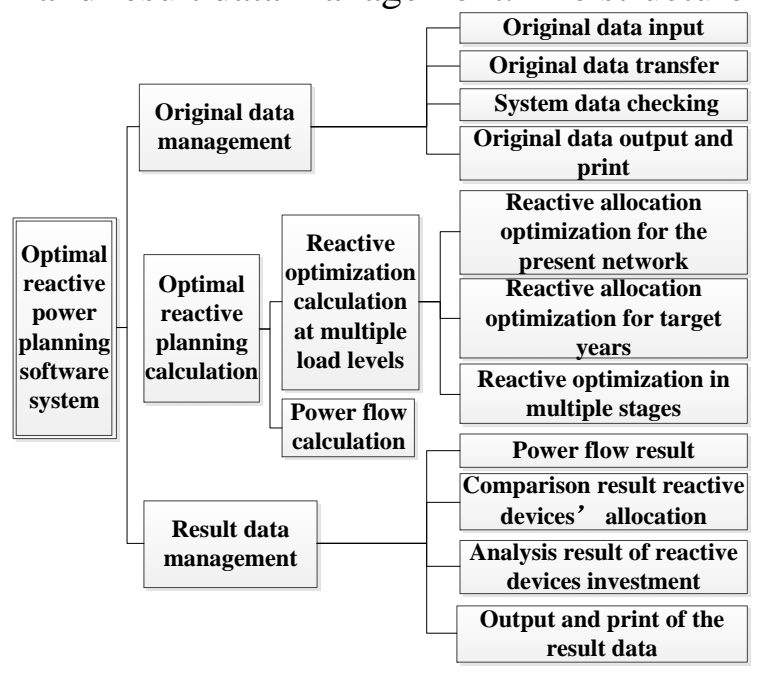

Fig. 3. Function structure of RPPSS

Module of Original Data Management. Except for the management and storage of the original data, the module also provides an excellent function of human-computer interaction in the data maintenance. Users can input, check, modify or output all the original data"visually" to ensure the optimal reactive calculating accuracy and the solutions' effectiveness.

As for the optimal reactive planning, it is probably incomplete for the basic data (data of network structure, load, or power source) which is transferred from the CNP's module of network structure. However, the complement, modification and maintenance of above data could be found in this module.

Otherwise, the module of original data management also provides a function of data checking, which is based on the related regulations (such as the planning directives), for the users to avoid inputting the incorrect data.

Module of Result Data Management. The result of the optimal reactive planning involves the node voltage information, the power flow information of the transmission lines, the output information of the power source point, the scale of reactive devices allocation and the financial comprehensive analysis. The module's main function is to manage this information, while the detailed function includes the power flow results, the comparison results of reactive devices' allocation, the analysis results of reactive devices investment and the input and print of the result data.

Module of Optimal Reactive Planning Calculation. The function of the module is divided into two parts: optimal reactive calculation at different load levels and the power flow calculation. The module mainly realizes the reactive allocation optimization for the present or target years' network, as well as the reactive optimization in multiple stages. Details as follows:

1) Reactive allocation optimization for the present network: According to the optimal reactive calculation of the present network, the software can obtain the optimal compensation allocations for the present network, the power flow before and after the compensation, the network loss result and the investment of reactive devices.

2) Reactive allocation optimization for target years: According to the result of network structure optimization and load forecasting, the software can optimize the compensation allocations, and consequently obtain the optimal locations and capacity of compensators for the target years and the result of power flow and network loss before and after the compensation. 
3) Reactive optimization in multiple stages: Differing from the reactive optimization in single stage, the multi-stage reactive optimization needs to combine with the present and the planning network structure, as well as the load development and the developing process of power network. Considering the present and planning reactive source allocations, the optimization applies the quasi-dynamic planning method to obtain the optimal compensation results that satisfy all the stages (one by one from the present to the target year) of the network development and furthermore provides reference for the network investment decision.

In the above procedure of the function realization, the automatic call the reactive optimization algorithm through the standardized interface is the core of this function module. As a part of OPF, the reactive optimization belongs to the nonlinear planning optimization with the characteristics of dynamic, uncertain, multiple targets and limits. The algorithm involves several aspects, such as the choice of reactive compensation location, the determination of reactive capacity, the control of transformer tap and the adjustment of generator terminal voltage. And the solving method mainly focuses on three aspects: the treatment of nonlinear function, the algorithm convergences, and the solutions to deal with discrete variables. As there are lots of algorithms for the optimal reactive planning, the general calculation is designed to combine the advantages of all the algorithms. The diagram is shown as Fig. 4, in which, except for Bender's decomposition method, the other heuristic algorithms [7,8,9,10,11] are involved, such as the genetic algorithm and the particle swarm optimization.

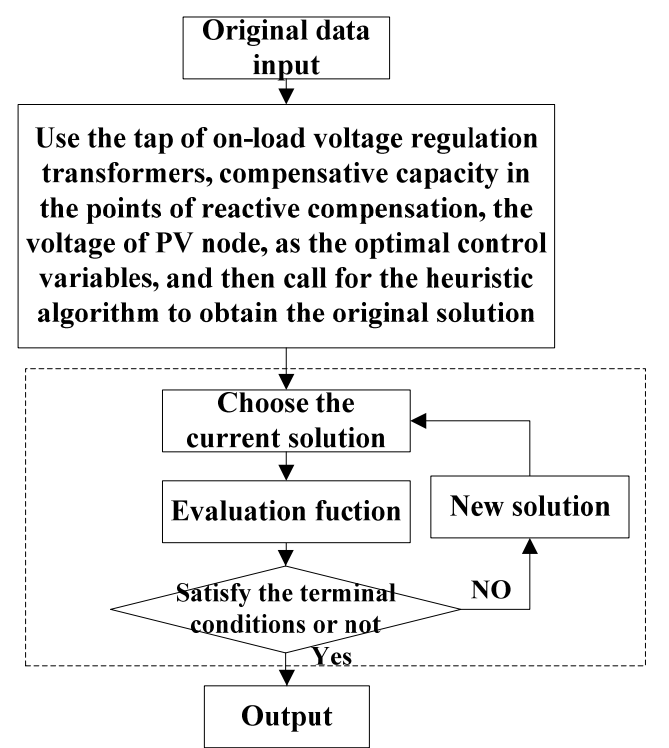

Fig.4. General calculation diagram for ORPP

For a certain load level or operating mode, the power flow calculation function can output the relevant power flow results. That is the basis of optimal iterations. Furthermore, considering the influence of different load levels, the software turns to support the reactive compensation calculation at different load levels. In this way, users are able to either get the relevant result according to the certain load level, or integrate all the results at different load levels to get an optimal one.

\section{Applications}

The optimal reactive planning software system developed in the paper is applied to achieve the planning of the high-voltage distribution network of a certain domestic area. The planning sets the initial year at 2000, and the target years at 2010 and 2020. The network scale is as follows: 130 nodes and 140 branches in 2000, 175 nodes and 235 branches in 2010, 179 nodes and 275 branches in 2020. According to actual condition, invest $80 \mathrm{RMB}$ in capacitor per $\mathrm{kVar}$ that is allocated at the $220 \mathrm{kV}$ bus, while $60 \mathrm{RMB}$ in the capacitor at $66 \mathrm{kV}$ bus. The power factor of generators of the local power plant takes values from 0.6 to 0.85 .

The constraint conditions input in the optimal calculation include: 
1) The allowable deviation of the source point voltage: $220 \mathrm{kV}$ and $66 \mathrm{kV}$ bus voltage is $-3 \% \sim+7 \%$ while $0 \% \sim+7 \%$ for the $10 \mathrm{kV}$ bus voltage;

2) The power factor of the public substation $\cos \varphi=0.85$, while for the specific substation $\cos \varphi=0.9$;

3) For the $220 \mathrm{kV}$ substation, the upper limit of the capacitor capacity at reactive compensation points is $25 \%$ of the main transformers' capacity, while $20 \%$ for the $66 \mathrm{kV}$ substation.

Take the 2010's optimal reactive planning results and relevant device allocations for example to explain the function of the software (as shown in Table 1).

Table 1. Results of reactive power optimization

\begin{tabular}{|c|c|c|c|c|c|c|}
\hline \multicolumn{2}{|c|}{ Node Number } & 20 & \multicolumn{2}{|c|}{$\begin{array}{c}\text { Investment of Reactive } \\
\text { Devices } \\
\end{array}$} & \multicolumn{2}{|c|}{0.0768} \\
\hline \multicolumn{2}{|c|}{$\begin{array}{l}\text { Original Capacity of } \\
\text { Reactive Devices }\end{array}$} & 20040.00 & $\begin{array}{r}\text { Result Capacity o } \\
\text { Devices }\end{array}$ & eactive & 2100 & \\
\hline $\begin{array}{c}\text { Serial } \\
\text { Number }\end{array}$ & Node Number & Node Title & $\begin{array}{l}\text { Original } \\
\text { Capacity }\end{array}$ & \multicolumn{2}{|c|}{ Result Capacity } & ... \\
\hline 1 & 13 & F-W Bus 63kV & 40080.00 & \multicolumn{2}{|c|}{40080.00} & $\ldots$ \\
\hline 2 & 14 & B-E Bus 63kV & 40560.00 & \multicolumn{2}{|c|}{400560.00} & $\ldots$ \\
\hline 3 & 16 & B-N Bus 63kV & 20040.00 & \multicolumn{2}{|c|}{21000.00} & $\ldots$ \\
\hline 4 & 19 & \multirow[t]{2}{*}{ No.9 Sub 63kV } & 0.00 & \multicolumn{2}{|c|}{0.00} & $\ldots$ \\
\hline$\ldots$ & $\ldots$ & & $\ldots$ & & & \\
\hline \multicolumn{2}{|c|}{$\begin{array}{c}\text { Total Original Reactive } \\
\text { Capacity }\end{array}$} & 447499.00 & \multicolumn{2}{|c|}{$\begin{array}{l}\text { Total Result Reactive } \\
\text { Capacity }\end{array}$} & \multicolumn{2}{|c|}{880640.00} \\
\hline \multicolumn{3}{|c|}{ Unit of investment(million RMB) } & \multicolumn{4}{|c|}{ Unit of capacity of reactive devices(kVar) } \\
\hline
\end{tabular}

The above table (Table 1) intuitively shows comparison of the reactive investment and device expenses of each bus and substation before and after the reactive compensation, and gives an intuitive reference to the network planning personnel.

Due to numerous nodes and the complication of the network, limited by space, only the economic analysis of reactive power optimization before and after is listed in Table 2 to show the optimal results and the compensation effects.

Table 2. Economic analysis of reactive power optimization

\begin{tabular}{|c|c|c|}
\hline Index & Before compensation & After compensation \\
\hline Lowest voltage(p.u.) & 0.8309 & 0.9701 \\
\hline Network loss(MW) & 0.1876 & 0.1242 \\
\hline Network loss rate(\%) & 1.1698 & 0.7750 \\
\hline $\begin{array}{c}\text { Network loss expense } \\
\text { (RMB per hour) }\end{array}$ & 6260.22 & 4146.88 \\
\hline Investment expense(million RMB) & & 0.6236 \\
\hline Annual profit(million RMB) & & 10.5614 \\
\hline
\end{tabular}

Table 2 shows that the optimized compensation, to some extent, can improve the voltage quality and the network loss levels, and consequently increases the economic benefit.

For the local power plant, the software shows relevant active and reactive power output as the reference for planning. In Table 3, the information of partial generators' output and power factors is listed. 
Table 3. Comparison of the power generation results before and after the reactive power compensation

\begin{tabular}{|c|c|c|c|c|c|}
\hline \multirow{2}{*}{ Generator } & \multicolumn{2}{|c|}{ Before compensation } & \multicolumn{3}{|c|}{ After compensation } \\
\cline { 2 - 6 } & Active (p.u) & $\begin{array}{c}\text { reactive } \\
(\boldsymbol{p . u})\end{array}$ & Active (p.u) & Reactive (p.u) & $\begin{array}{c}\text { Power } \\
\text { factor }\end{array}$ \\
\hline $\begin{array}{c}500 \mathrm{kV} \\
\text { Ballance bus }\end{array}$ & 15.314 & 8.3599 & 15.2518 & 7.7391 & 0.8918 \\
\hline Bus 1 & 0.4030 & 1.9301 & 0.4030 & 0.4413 & 0.6743 \\
\hline Bus 2 & 0.2560 & 1.1931 & 0.2556 & 0.1811 & 0.8163 \\
\hline Bus 3 & 0.1202 & 0.6104 & 0.1197 & 0.1118 & 0.7305 \\
\hline$\ldots .$. & $\ldots$ & $\ldots$ & $\ldots$ & $\ldots$ & $\ldots$ \\
\hline
\end{tabular}

The optimal result suggests that the optimal reactive planning software not only improves or voltage quality and decrease the network loss, but also satisfies the power factor requirement for the local power plant.

Vertically compared with the optimal results of different planning years, the optimal reactive planning module also shows reactive power optimization in the multiple stages, as is shown in Table 4.

Table 4. Comparison of multi-stage reactive power optimization

\begin{tabular}{|c|c|c|c|c|}
\hline \multicolumn{2}{|c|}{ Index } & \multicolumn{3}{c|}{ Planning year } \\
\cline { 3 - 5 } & & $\mathbf{2 0 0 0}$ & $\mathbf{2 0 1 0}$ & $\mathbf{2 0 2 0}$ \\
\hline \multirow{2}{*}{ Network loss rate (\%) } & Before & 1.3013 & 1.3052 & 1.1698 \\
\cline { 2 - 5 } & After & 0.8930 & 0.8717 & 0.7750 \\
\hline \multirow{2}{*}{ Lowest voltage (p.u) } & Before & 0.8678 & 0.8493 & 0.8309 \\
\cline { 2 - 5 } & After & 0.9702 & 0.9701 & 0.9701 \\
\hline
\end{tabular}

As is demonstrated in the Table.4, multi-stage reactive power optimization is able to likewise satisfy the demand of load development in the planning area, and avoid overlapping investment of the reactive devices.

\section{Summary}

Considering the characteristics of the optimal reactive power planning and its requirements in practice, the reactive power optimization software that can satisfy the practical requirement is developed in the paper. The following is the characteristics of RPPSS:

1) The software is quite effective for the practical works. It is able to achieve the optimal reactive planning both in a single stage and in multiple stages. Moreover, with its assist, the calculation for the optimal reactive allocation becomes effectively automatic and in consequence it relieves the burden of the staffs of the network planning.

2) The software, in the one hand, can be embedded as a subsystem into CNP to accomplish the whole works of the network planning. And in the other hand, it can be applied as an independent software system to achieve all the tasks of reactive planning.

3) The software combines the heuristic algorithm with Bender's decomposition for an effective calculation. Otherwise, due to the layered structure, the software is of an excellent scalability for its convenience to upgrade.

\section{Acknowledgements}

This work was financially supported in part by the National Natural Science Foundation of China (No. 51207101), China Postdoctoral Science Foundation (No. 2013M530113), and the Science and Technology Projects of State Grid Corporation of China (No. kjgw2014-009). 


\section{References}

[1] Yixin Yu, Chengshan Wang and Jun Xiao, et al.: Automation of Electric Power Systems. Vol. 24(2000), p. 59-62 (In Chinese).

[2] Chengshan Wang and Yi Zhang: Proceeding s of the CSU-EPSA. Vol. 15(2003), p. $46-50$ (In Chinese).

[3] Chengshan Wang, Xiaoli Tang and Yixin Yu, et al.: Automation of Electric Power Systems. Vol. 22(1998), p. 14-17 (In Chinese).

[4] Chengshan Wang, Xiaoli Tang and Yixin Yu: Power System Technology. Vol. 22(1998), p. 23-26 (In Chinese).

[5] Ting Cui, Yuanzhang Sun and Jian Xu, et al.: Proceedings of the CSEE. Vol. 31(2011), p. 43-50 (In Chinese).

[6] Kun Zhao and Guangfei Geng: Power System Protection and Control. Vol. 35(2011). p. 57-62 (In Chinese).

[7] Hong Liu, Shaoyun Ge and Hui Li: Journal of Tianjin University. Vol. 42(2009), p. 796-801 (In Chinese).

[8] Maoya Shen, Xiaoqun Ding and Zhongda Wang, et al.: Proceedings of the CSU-EPSA. Vol. 19(2007), p. 84-87 (in Chinese).

[9] Jun Xiao, Bo Wang and Chengshan Wang, et al.: Distribution \& Utilization. Vol. 25(2008), p. 21-24 (In Chinese).

[10] Yixin Yu, Chengshan Wang and Shaoyun Ge, et al.: Transactions of Tianjin University, vol. 10(2004) (In Chinese).

[11] Fengzhang Luo, Chengshan Wang and Jun Xiao, et al.: Power System Technology. Vol. 33(2009), p. 79-83 (In Chinese).

[12] Feng Li, Zhengchao Liu and Fengzhang Luo, et al.: Proceedings of the CSU-EPSA. Vol. 23(2011), p. 151-156 (In Chinese). 